\title{
Comparison of the effects of sertraline and L-carnitine on intradialytic hypotension; a double blind clinical trial
}

\author{
Sanaz Jamshidi ${ }^{1 \oplus}$, Sepideh Hajian $^{2^{*}}$, Nafiseh Rastgoo $^{1^{\circledR}}$, Navid Mohammadi $^{3}{ }^{\circledR}$ \\ ${ }^{1}$ Qazvin University of Medical Sciences, Qazvin, Iran \\ ${ }^{2}$ Department of Nephrology, Velayat Hospital, Qazvin University of Medical Sciences, Qazvin, Iran \\ ${ }^{3}$ Department of Family and Community Medicine, School of Medicine, Qazvin University of Medical Sciences, Qazvin, Iran
}

\section{A R T I C L E I N F O}

\section{Article Type:}

Original

\section{Article History:}

Received: 10 January 2020

Accepted: 4 March 2020

Published online: 28 March 2020

\section{Keywords:}

Hemodialysis

Intradialytic hypotension

Sertraline

L-Carnitine

Chronic kidney disease

End-stage kidney disease

\begin{abstract}
ABS T RACT
Introduction: Although some studies have reported the positive effect of sertraline and L-carnitine on intradialytic hypotension (IDH), a common complication of dialysis, however the results are controversial.

Objectives: The aim of this study was to compare the effects of sertraline and L-carnitine on blood pressure in patients with chronic renal failure who were undergoing dialysis.

Patients and Methods: This double-blind clinical trial was conducted on 32 hemodialysis patients who suffered from IDH in more than $50 \%$ of dialysis sessions. Patients were randomly divided into two groups of sertraline (50 mg daily) and L-carnitine (1000 mg daily), with 16 patients in each group. Duration of treatment was four weeks, then patients were followed up for additional three weeks. The changes in patients' blood pressure were monitored in each group and the results compared between the two groups.

Results: Of all, 18 patients (56\%) were female, 14 patients (44\%) were male, and their mean (SD) age was $60 \pm 15$ years. At the end of the study, mean systolic blood pressure (SBP), mean diastolic blood pressure (DBP), and mean arterial pressure (MAP) were significantly increased in both the sertraline and L-carnitine groups $(P<0.05)$. In addition, nadir SBP, nadir DBP, and nadir MAP in each group were significantly increased compared to pre-treatment period $(P<0.001)$. An increase of more than $5 \mathrm{~mm} \mathrm{Hg}$ in SBP, DPB, and MAP was observed in half of the subjects in the sertraline group and more than two-thirds of the patients in the L-carnitine group, however there was no significant difference between the two groups $(P>0.05)$.

Conclusion: The findings of this study showed that the administration of sertraline or L-carnitine for one month could significantly increase SBP, DBP, MAP, and nadir blood pressures in dialysis patients suffering from IDH during dialysis sessions because there was no significant difference between the two drugs.

Trial Registration: The trial protocol has been registered by the Iranian registry of clinical trial (IRCT20190624043997N1; https://irct.ir/trial/40941, ethical code \# IR.QUMS.REC.1397.128).
\end{abstract}

Implication for health policy/practice/research/medical education:

In a double blind clinical trial on 32 hemodialysis patients who suffered from intradialytic hypotension, in more than $50 \%$ of dialysis sessions, we found that the administration of sertraline or L-carnitine for one month could significantly increase the mean systolic blood pressure, mean diastolic blood pressure, and mean arterial pressure and also nadir blood pressures while there was no significant difference between both drugs.

Please cite this paper as: Jamshidi S, Hajian S, Rastgoo N, Mohammadi N. Comparison of the effects of sertraline and L-carnitine on intradialytic hypotension; a double blind clinical trial. J Renal Inj Prev. 2020; 9(3): e22. doi: 10.34172/jrip.2020.22.

\section{Introduction}

Although dialysis is an effective method for the management of end-stage kidney disease (ESKD), it is associated with some complications, including intradialytic hypotension (IDH). There is no consensus regarding the definition of IDH, but the Disease Outcomes Quality Initiative (KDOQI) defines this condition as a decrease of $20 \mathrm{~mm}$
$\mathrm{Hg}$ or more in systolic blood pressure (SBP) or a decrease of $10 \mathrm{~mm} \mathrm{Hg}$ or more in mean arterial pressure (MAP) requiring therapeutic interventions (1). The incidence of symptomatic hypotension during or immediately after dialysis is reported in 5\%-30\% of all dialysis sessions $(2,3)$. Moreover, some patients experience IDH in more than $50 \%$ of their dialysis sessions (4). 
IDH is usually associated with different symptoms such as dizziness, muscle cramps, abdominal discomfort, nausea, vomiting, yawning and sighing (3). Repeated episodes of IDH are associated with increased morbidity and morbidity in patients $(5,6)$ and may decrease the effects of dialysis because such a condition may make it necessary to modify treatment and stop dialysis. In severe cases, it can be associated with intestinal ischemia, stroke or myocardial infarction and fistula thrombosis $(3,7)$. IDH usually occurs in the absence of serious medical monitoring and may emerge because of several factors such as rapid or excessive ultrafiltration, rapid decrease in plasma osmolarity, incorrectly low-prescribed target weight, autonomic neuropathy, and use of pre-dialysis anti-hypertensive medications or meals during the dialysis session, and also the composition and temperature of the dialysate fluid $(2,3,8)$.

To manage and treat IDH, it may become necessary to alter or stop ultrafiltration, place patients in a Trendelenburg position, administer oxygen, administration of intravenous fluids, and evaluate the serious medical conditions (9). However, patients with recurrent IDH episodes may need preventive interventions. For instance it might be necessary to modify ultrafiltration rate and re-assess the appropriate target weight $(10,11)$, use of cold dialysate (12), change the dialysis frequency and duration (13), and evaluate patients to detect acute medical conditions such as underlying cardiac problems or hemolysis (14).

So far, few drugs have been recommended to prevent $\mathrm{IDH}$. The use of midodrine is effective in the prevention of IDH (15), but it can cause complications such as pruritus, supine hypotension, urinary retention, and pilomotor reactions that limit its use (16). Sertraline, a selective serotonin reuptake inhibitor (SSRI), recently used to prevent IDH. However, a small number of studies have been conducted in this area (17-20) and their results are contradictory. In addition, L-carnitine, an important cofactor required for smooth muscle and heart muscle, has been used in dialysis patients since some studies have indicated its positive effect on IDH $(21,22)$. However, some studies have failed to prove its effect (23). Thus, routine use of these two drugs for the prevention of IDH has not been recommended yet.

\section{Objectives}

The present study was designed to compare the effect of the two drugs of sertraline and L-carnitine on changes in blood pressure in patients with chronic kidney disease undergoing dialysis.

\section{Patients and Methods \\ Study design}

This double blind clinical trial was conducted on hemodialysis patients admitted to Bou Ali and Velayat hospitals in Qazvin, Iran in 2019. Using census sampling method, from 38 patients, a total of 32 patients undergoing dialysis for at least two months were enrolled in the study (Figure 1). The selected subjects had not recently used a blood pressure lowering medication. The patients using bicarbonate solution for dialysis, suffered from

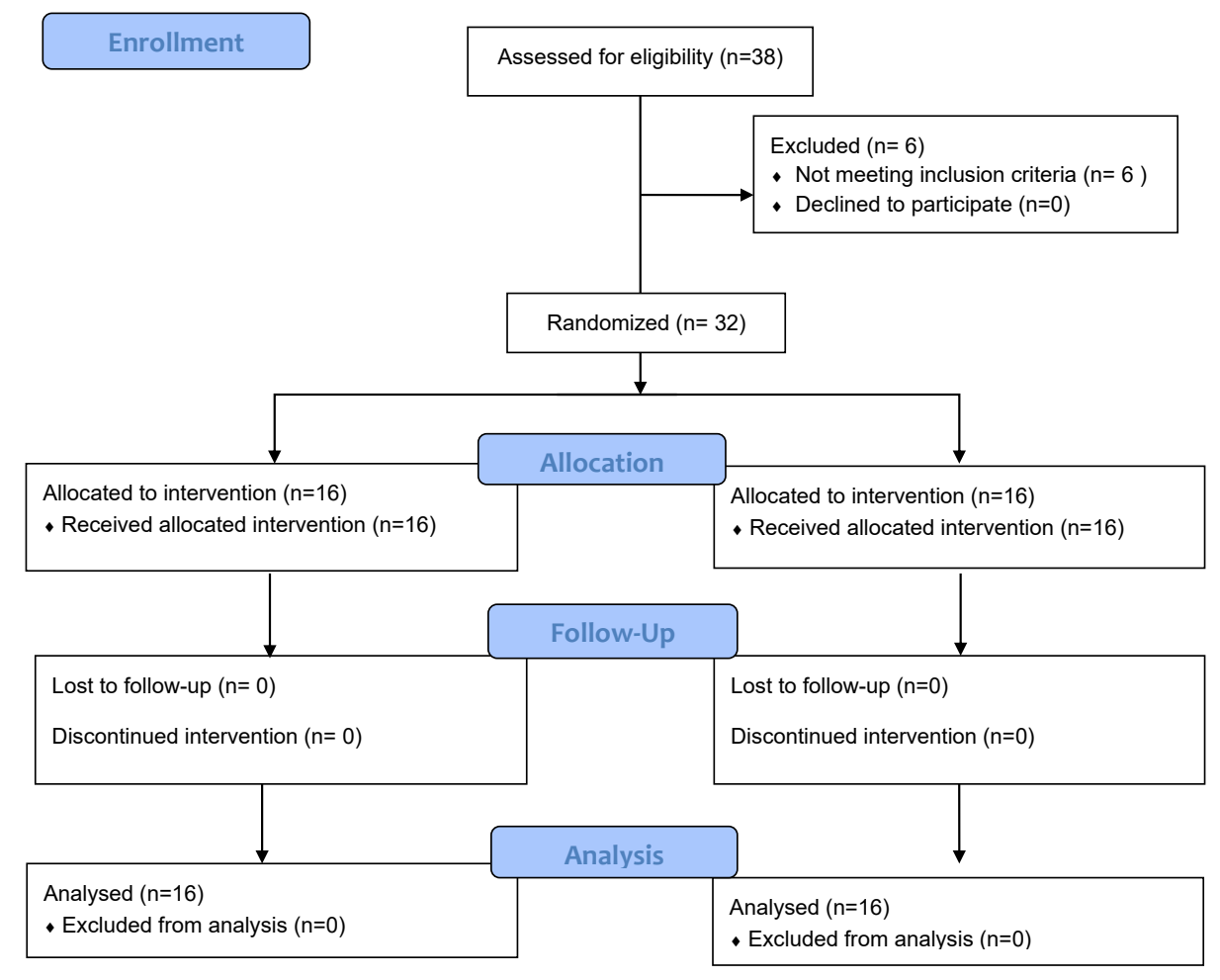

Figure 1. Flow diagram of the study. 
hypotension during dialysis in more than $50 \%$ of dialysis sessions, and were willing to participate in the study. IDH is defined as a decrease of $20 \mathrm{~mm} \mathrm{Hg}$ or more in SBP or a decrease of $10 \mathrm{~mm} \mathrm{Hg}$ or more in MAP during dialysis session (1).

Initially, the research team recorded the data of patients including age, gender, height, weight, body mass index (BMI), diabetes status, presence of hypotension at home, duration of dialysis, number of dialysis sessions per week, type of vascular access, type of filter, midodrine administration, and the various laboratory results (including serum calcium, iron, total iron binding capacity [TIBC], ferritin, intact PTH, Kt/V, blood urea nitrogen [BUN] and creatinine). In addition, nadir blood pressures (SBP, diastolic blood pressure [DBP], and MAP) were extracted from dialysis charts since four weeks before treatment. Patients' SBP and DBP were measured before, during, and after the first dialysis session since mean values were recorded as the baseline values. In addition, MAP calculated using the following formula:

$$
M A P=D B P+(S B P-D B P) / 3
$$

Using random number table, the patients then were randomly divided into the sertraline group (50 mg daily, Sobhan Daru Co., Iran) and L-carnitine group (1000 mg daily, Perarin Pars Co., Iran), with 16 members in each group (Figure 1). The appearances of the pills were the same since patients received the drug for free. Both the patients and the clinical caregivers were unaware of groups; thus, the study was double-blind. The patients were treated for four weeks and followed up for additional three weeks. At the end of the study, blood pressure was measured before, during, and after the last dialysis session and their mean values were recorded. In addition, nadir blood pressures were recorded during treatment and follow-up period. An increase of $5 \mathrm{~mm} \mathrm{Hg}$ in blood pressure, as compared with the baseline, was considered as an effect of the drug and compared between the two groups.

\section{Ethical issues}

The research followed the tenets of the Declaration of Helsinki. The Ethics Committee of Qazvin University of Medical Sciences approved this study (IR.QUMS. REC.1397.128). The institutional ethical committee at Qazvin University of Medical Sciences approved all study protocols. The study was registered as a clinical trial at the Iranian Registry of Clinical Trials website (IRCT20190624043997N1; https://irct.ir/trial/40941). Accordingly, written informed consent was taken from all participants before any intervention.

\section{Statistical analysis}

SPSS 25 software was used for data analysis. Qualitative variables were described using frequency and percentage and quantitative variables were presented using means and standard deviation. Chi-square test, independent $t$ test and paired $t$ test were used for data analysis. $P<0.05$ was set as the level of significance.

\section{Results}

Of all, 18 patients (56\%) were female and 14 patients (44\%) were male and their mean age was $60 \pm 15$ years. Mean (SD) BMI in all patients was $25.7 \pm 5.8 \mathrm{~kg} / \mathrm{m}^{2}$. Moreover, four patients (13\%) had diabetes and 10 patients (31\%) had hypotension at home. There was no significant difference between patients in the sertraline and L-carnitine groups regarding age, gender, height, weight, BMI, diabetes, and hypotension at home (Table 1). All the patients were undergoing three dialysis sessions per week while mean

Table 1. Comparison of patient characteristics between two groups

\begin{tabular}{|c|c|c|c|c|}
\hline & & Sertraline group $(n=16)$ & L-carnitine group ( $n=16)$ & $P$ value \\
\hline \multirow{2}{*}{ Gender } & Male & $7(44 \%)$ & $7(44 \%)$ & \multirow{2}{*}{$1.000 *$} \\
\hline & Female & $9(56 \%)$ & $9(56 \%)$ & \\
\hline \multicolumn{2}{|l|}{ Age, year } & $61 \pm 17$ & $59 \pm 13$ & $0.677^{* *}$ \\
\hline \multicolumn{2}{|l|}{ Weight, kg } & $65 \pm 18$ & $73 \pm 13$ & $0.163^{* *}$ \\
\hline \multicolumn{2}{|l|}{ Height, cm } & $161 \pm 6$ & $167 \pm 8$ & $0.051 * *$ \\
\hline \multicolumn{2}{|l|}{$\mathrm{BMI}, \mathrm{kg} / \mathrm{m}^{2}$} & $24.9 \pm 6.5$ & $26.4 \pm 5.0$ & $0.474 * *$ \\
\hline \multicolumn{2}{|l|}{ Diabetes } & $1(6 \%)$ & $3(19 \%)$ & $0.258 * *$ \\
\hline \multicolumn{2}{|c|}{ Hypotension at home } & $5(31 \%)$ & $5(31 \%)$ & $1.000 *$ \\
\hline \multicolumn{2}{|c|}{ Duration of dialysis, year } & $4.6 \pm 3.7$ & $4.7 \pm 2.0$ & $0.953 * *$ \\
\hline \multirow{3}{*}{ Vascular access } & AVF & $9(56 \%)$ & $14(88 \%)$ & \multirow{3}{*}{$0.037^{*}$} \\
\hline & Catheter & 7 (44\%) & $1(6 \%)$ & \\
\hline & Graft & $0(0 \%)$ & $1(6 \%)$ & \\
\hline \multirow{2}{*}{ Type of filter } & Low flux & $10(63 \%)$ & $6(37 \%)$ & \multirow{2}{*}{$0.157^{*}$} \\
\hline & High flux & $6(37 \%)$ & $10(63 \%)$ & \\
\hline \multicolumn{2}{|c|}{ Midodrine intake } & $3(19 \%)$ & $2(13 \%)$ & $0.626^{*}$ \\
\hline
\end{tabular}

BMI, Body mass index; AVF, arterial venous fistula.

* Chi-square test, $* *$ independent sample $t$ test. 
(SD) duration of dialysis for all the patients was $4.7 \pm 2.9$ years. Concerning vascular access, 23 patients (72\%) had arterial venous fistula, eight patients (25\%) had catheters, and one patient $(3 \%)$ had graft. Half of the patients used low-flux filter and the other half used high-flux filter. Of all, five patients (16\%) were taking midodrine. Regarding vascular access, the sertraline group had more catheters than the L-carnitine group $(P=0.037)$. There was no significant difference between the two groups in terms of other dialysis characteristics such as duration of dialysis, type of filter, and administration of midodrine (Table 1). Table 2 presents the results of the patients' laboratory tests. As the table depicts, the comparison of the results between the two groups at baseline showed no significant difference $(P>0.05)$.

Mean (SD) of SBP in all the patients at baseline and after the intervention was $82 \pm 7 \mathrm{~mm} \mathrm{Hg}$ and $100 \pm 15 \mathrm{~mm} \mathrm{Hg}$, respectively, which was significantly increased $(P<0.001)$. Mean (SD) of DBP in all the patients at baseline and after the intervention was $52 \pm 7 \mathrm{~mm} \mathrm{Hg}$ and $66 \pm 11 \mathrm{~mm} \mathrm{Hg}$, respectively, which was significantly increased $(P<0.001)$. Mean (SD) MAP in all the patients at baseline and after the intervention was $66 \pm 6 \mathrm{~mm} \mathrm{Hg}$ and $77 \pm 12 \mathrm{~mm} \mathrm{Hg}$, respectively, which was significantly increased $(P<0.001)$ too. Mean SBP, DBP, and MAP in both groups significantly increased after the intervention (Table 3). An increase of more than $5 \mathrm{~mm} \mathrm{Hg}$ in SBP, DBP and MAP was observed

Table 2. Comparison of patient laboratory test results between the two groups

\begin{tabular}{lccc}
\hline & $\begin{array}{c}\text { Sertraline group } \\
(\mathbf{n}=\mathbf{1 6})\end{array}$ & $\begin{array}{c}\text { L-carnitine group } \\
(\mathbf{n}=\mathbf{1 6})\end{array}$ & $\begin{array}{c}\boldsymbol{P} \\
\text { value* }\end{array}$ \\
\hline Calcium, mg/dL & $8.8 \pm 1.8$ & $8.4 \pm 1.5$ & 0.524 \\
Iron, $\mu \mathrm{g} / \mathrm{dL}$ & $55 \pm 17$ & $58 \pm 18$ & 0.630 \\
TIBC, $\mu \mathrm{g} / \mathrm{dL}$ & $336 \pm 71$ & $310 \pm 58$ & 0.271 \\
Ferritin, $\mathrm{ng} / \mathrm{mL}$ & $655 \pm 345$ & $595 \pm 409$ & 0.662 \\
$\mathrm{iPTH}, \mathrm{pg} / \mathrm{mL}$ & $397 \pm 245$ & $486 \pm 589$ & 0.582 \\
$\mathrm{Kt} / \mathrm{V}$ & $1.19 \pm 0.31$ & $1.06 \pm 0.20$ & 0.186 \\
BUN, $\mathrm{mg} / \mathrm{dL}$ & $65 \pm 12$ & $59 \pm 18$ & 0.328 \\
Creatinine, $\mathrm{mg} / \mathrm{dL}$ & $10.04 \pm 2.81$ & $9.31 \pm 2.88$ & 0.477 \\
\hline
\end{tabular}

iPTH, intact PTH; TIBC, total iron binding capacity; BUN, blood urea nitrogen.

* Independent sample $t$ test.

Table 3. Comparison of SBP, DBP, and MAP before and after study in each group

\begin{tabular}{lllll}
\hline & & Before & After & P value* \\
\hline \multirow{2}{*}{ Sertraline group } & SBP, mm Hg & $83 \pm 8$ & $99 \pm 15$ & 0.003 \\
(n=16) & DBP, mm Hg & $56 \pm 5$ & $67 \pm 10$ & 0.003 \\
& MAP, mm Hg & $65 \pm 5$ & $77 \pm 12$ & $<0.001$ \\
L-carnitine group & SBP, mm Hg & $81 \pm 6$ & $102 \pm 15$ & $<0.001$ \\
(n=16) & DBP, mm Hg & $49 \pm 6$ & $65 \pm 13$ & $<0.001$ \\
& MAP, mm Hg & $59 \pm 5$ & $77 \pm 13$ & $<0.001$ \\
\hline
\end{tabular}

DBP, diastolic blood pressure; MAP, mean arterial pressure; SBP, systolic blood pressure.

* Paired $t$ test. in 20 (63\%), 18 (56\%), and 19 (59\%)patients, respectively, since no significant difference between the two groups was detected (Table 4). The nadir blood pressures in each group were significantly increased too (Table 5).

There were five cases of nausea and two cases of mood improvement in the sertraline group. There were four cases of mood improvements in the L-carnitine group too.

\section{Discussion}

The findings of this study showed that the administration of sertraline or L-carnitine for one month could significantly increase SBP, DBP, and MAP in dialysis patients suffering from IDH during dialysis sessions while there was no significant difference between two drugs.

The exact mechanism of IDH is unclear, however, the abrupt withdrawal of sympathetic vasoconstrictor tone at the late stages of dialysis ultrafiltration is identified as one of the factors contributing to IDH, which can cause a sudden drop in blood pressure and results in subsequent hypotension (24). Other conditions such as neurocardiogenic syncope, idiopathic orthostatic hypotension, and carotid sinus hypersensitivity are triggered by the abrupt withdrawal of sympathetic vasoconstrictor tone, too (25-27). These responses seem to be due to a sudden surge in serotonin concentration in some parts of the central nervous system (CNS) that may be controlled by serotonergic receptor block (28). Therefore, some SSRIs are used to treat neurocardiogenic syncope and idiopathic orthostatic hypotension $(26,27,29,30)$. Furthermore, fluoxetine is used to treat

Table 4. Comparison of the frequency of increased blood pressure greater than $5 \mathrm{~mm} \mathrm{Hg}$ between the two groups

\begin{tabular}{lccc}
\hline & $\begin{array}{c}\text { Sertraline group } \\
(\mathbf{n}=\mathbf{1 6})\end{array}$ & $\begin{array}{c}\text { L-carnitine group } \\
(\mathbf{n}=16)\end{array}$ & $\begin{array}{c}\boldsymbol{P} \\
\text { value* }\end{array}$ \\
\hline SBP & $8(50 \%)$ & $12(75 \%)$ & 0.144 \\
DBP & $8(50 \%)$ & $10(63 \%)$ & 0.476 \\
MAP & $8(50 \%)$ & $11(69 \%)$ & 0.280 \\
\hline
\end{tabular}

DBP, diastolic blood pressure; MAP, mean arterial pressure; SBP, systolic blood pressure.

* Chi square test.

Table 5. Comparison of nadir blood pressure before and after study in each group

\begin{tabular}{llccc}
\hline & & Before & After & P value* \\
\hline \multirow{2}{*}{$\begin{array}{l}\text { Sertraline } \\
\text { group (n=16) }\end{array}$} & Nadir SBP, mm Hg & $73 \pm 8$ & $87 \pm 14$ & 0.004 \\
& Nadir DBP, mm Hg & $47 \pm 7$ & $57 \pm 10$ & 0.005 \\
& Nadir MAP, mm Hg & $56 \pm 6$ & $66 \pm 10$ & 0.002 \\
L-carnitine & Nadir SBP, mm Hg & $68 \pm 8$ & $86 \pm 15$ & $<0.001$ \\
group (n=16) & Nadir MAP, mm Hg & $41 \pm 7$ & $56 \pm 13$ & 0.002 \\
& & $49 \pm 6$ & $66 \pm 13$ & $<0.001$ \\
\hline
\end{tabular}

DBP, diastolic blood pressure; MAP, mean arterial pressure; SBP, systolic blood pressure.

* Paired $t$ test. 
postural hypotension during hemodialysis too (31).

Sertraline, as an SSRI, is widely used for the treatment of depression in dialysis patients because it has appropriate pharmacodynamics and pharmacokinetics properties, good level of tolerability, low level of drug interactions, high level of safety, and low-cost (32). So far, few studies have investigated the effect of sertraline on IDH. Dheenan et al conducted a retrospective study on nine dialysis patients suffering from IDH who received sertraline for depression treatment. They compared the data collected six weeks before the treatment with the data collected six weeks after the treatment. They found, after the administration of sertraline, nadir MAP was increased to $13 \mathrm{~mm} \mathrm{Hg}$. In addition, the number of episodes of hypotension in dialysis sessions and the need for interventions decreased significantly (17). Yalcin et al reported that SBP significantly increased in nine IDH patients after four weeks of treatment using 100 mg daily sertraline, however, the increase in DBP was not significant (18). In another study, the researchers reported that the administration of $50 \mathrm{mg}$ sertraline daily was effective in reducing IDH episodes. Since, its mechanism of action may be through the improvement of regulation of autonomic response to hypovolemia (20). However, Brewster et al reported that the administration of sertraline for patients with IDH, who were treated with cold dialysate and midodrine, had no extra effect on blood pressure improvement (33). However, in their study, a significant number of the patients had diabetes, and diabetes neuropathy which might be introduced as one of the causes of inhibiting the effect of sertraline. According to a most recent study by Molin et al, sertraline may reduce the symptoms of IDH and improve its needed interventions, but it has no significant effect on blood pressure. In that study, 9 out of 16 patients had diabetes (19). The discrepancy between the results of the abovementioned studies indicates that sertraline had positive effects on blood pressure or IDH. Our study showed that one-month treatment by sertraline was associated with a significant increase in SBP, DBP, MAP, and nadir blood pressures. In addition, in half of the patients, SBP, DBP, and MAP increased by five mmHg or more. However, in our study, only one patient had diabetes. Therefore, it seems that further studies with a larger sample size and more accurate methods of sampling are required. For example, if the rate and severity of neuropathy in diabetic patients is measured before the study, it may identify patients who benefit most from this type of treatment.

The other drug used in our study was L-carnitine. Carnitine is an important cofactor required for smooth muscle and cardiac muscle that is deficient in ESKD patients. Its deficiency causes various complications such as fatigue and weakness, muscle cramps, exacerbation of anemia, hypotension, and cardiomyopathy (22). Like sertraline, few studies have investigated the effect of
L-carnitine on IDH. Additionally, Ahmed et al found that L-carnitine, as compared with placebo, was associated with a decrease in IDH in hemodialysis patients. Its mechanism of action is unclear, however its positive effect may be attributed to improvements in smooth muscle and cardiac function (22). Conversely, the study by Lynch et al did not support the effect of L-carnitine on dialysisrelated hypotension (23). In a clinical trial in 2013, Kudoh et al investigated the effect of L-carnitine (900 mg daily) on cardiac function and hypotension episodes in dialysis patients for three months. As they reported, the number of hypotension episodes per month decreased significantly (from 4 to 1.3 per month) (21). Likewise, in a clinical trial, Ibarra-Sifuentes et al showed that the intravenous administration of L-carnitine just before each dialysis session effectively decreased hypotension episodes (34). Since the results of studies on the effect of L-carnitine on IDH are controversial, however, our study showed that, like sertraline, L-carnitine significantly increased SBP, DBP, MAP, and nadir blood pressures. In addition, in twothirds of the patients, SBP, DBP, and MAP were increased by five $\mathrm{mmHg}$.

In our study, the results of the comparison between sertraline and L-carnitine showed the effectiveness of both drugs in elevating blood pressure in hemodialysis patients since there was no significant difference between both groups. Therefore, both drugs seem to be useful for the treatment of patients undergoing dialysis who suffer from IDH episodes.

\section{Conclusion}

The findings of this study showed that the administration of sertraline or L-carnitine for one month could significantly increase SBP, DBP, MAP, and nadir blood pressures in dialysis patients suffering from IDH during dialysis sessions while there was no significant difference between the two drugs.

\section{Limitations of the study}

Our study limitations were: small sample size, single center study, and short treatment period. The use of medications in dialysis patients still requires extensive studies, with the larger sample size, multicenter designs, and longer periods to evaluate the efficacy and safety of these drugs.

\section{Authors' contribution}

All authors passed four criteria for authorship contribution based on recommendations of the International Committee of Medical Journal Editors. SJ, SH and NR designed the protocol of study. SJ, NR and NM developed the protocol and performed it. Critical revision of the manuscript for important intellectual content was performed by SJ, SH, and NR. Analysis of data performed by NR. All authors read and approved the final paper. 


\section{Conflicts of interest}

The authors declare that they have no conflicts of interest.

\section{Ethical considerations}

Ethical issues (including plagiarism, data fabrication, double publication) have been completely observed by the authors.

\section{Funding/Support}

This study was supported by Qazvin University of Medical Sciences (Grant\# 14003170).

\section{References}

1. Kooman J, Basci A, Pizzarelli F, Canaud B, Haage P, Fouque $\mathrm{D}$, et al. EBPG guideline on haemodynamic instability. Nephrol Dial Transplant 2007;22 Suppl 2:ii22-44. doi: 10.1093/ndt/gfm019.

2. Sands JJ, Usvyat LA, Sullivan T, Segal JH, Zabetakis P, Kotanko P, et al. Intradialytic hypotension: frequency, sources of variation and correlation with clinical outcome. Hemodial Int. 2014;18:415-22. doi: 10.1111/hdi.12138.

3. Reilly RF. Attending rounds: A patient with intradialytic hypotension. Clin J Am Soc Nephrol 2014;9:798-803. doi: 10.2215/CJN.09930913.

4. Santos SF, Peixoto AJ, Perazella MA. How should we manage adverse intradialytic blood pressure changes? Adv Chronic Kidney Dis 2012;19:158-65. doi: 10.1053/j. ackd.2012.03.003.

5. Shoji T, Tsubakihara Y, Fujii M, Imai E. Hemodialysisassociated hypotension as an independent risk factor for two-year mortality in hemodialysis patients. Kidney International 2004;66:1212-20. doi: 10.1111/j.15231755.2004.00812.x.

6. Flythe JE, Inrig JK, Shafi T, Chang TI, Cape K, Dinesh K, et al. Association of intradialytic blood pressure variability with increased all-cause and cardiovascular mortality in patients treated with long-term hemodialysis. Am J Kidney Dis. 2013;61:966-74. doi: 10.1053/j.ajkd.2012.12.023.

7. Chang TI, Paik J, Greene T, Desai M, Bech F, Cheung $\mathrm{AK}$, et al. Intradialytic hypotension and vascular access thrombosis. J Am Soc Nephrol. 2011;22:1526-33. doi: 10.1681/ASN.2010101119.

8. Flythe JE, Kunaparaju S, Dinesh K, Cape K, Feldman HI, Brunelli SM. Factors associated with intradialytic systolic blood pressure variability. Am J Kidney Dis. 2012;59:40918. doi: 10.1053/j.ajkd.2011.11.026.

9. Emili S, Black NA, Paul RV, Rexing CJ, Ullian ME. A protocol-based treatment for intradialytic hypotension in hospitalized hemodialysis patients. Am J Kidney Dis. 1999;33:1107-14. doi: 10.1016/S0272-6386(99)70148-4.

10. van de Pol AC, Frenken LA, Moret K, Baumgarten R, van der Sande FM, Beerenhout CM, et al. An evaluation of blood volume changes during ultrafiltration pulses and natriuretic peptides in the assessment of dry weight in hemodialysis patients. Hemodial Int. 2007;11:51-61. doi: 10.1111/j.1542-4758.2007.00154.x.

11. Agar JW. Personal viewpoint: limiting maximum ultrafiltration rate as a potential new measure of dialysis adequacy. Hemodial Int. 2016;20:15-21. doi: 10.1111/ hdi.12288.

12. Mustafa RA, Bdair F, Akl EA, Garg AX, Thiessen-Philbrook $\mathrm{H}$, Salameh $\mathrm{H}$, et al. Effect of lowering the dialysate temperature in chronic hemodialysis: a systematic review and meta-analysis. Clin J Am Soc Nephrol. 2016;11:442-57. doi: $10.2215 / C J N .04580415$.

13. Kotanko P, Garg AX, Depner T, Pierratos A, Chan CT, Levin NW, et al. Effects of frequent hemodialysis on blood pressure: Results from the randomized frequent hemodialysis network trials. Hemodial Int. 2015;19:386401. doi: $10.1111 /$ hdi. 12255

14. Nette RW, van den Dorpel MA, Krepel HP, Ie EH, van den Meiracker AH, Poldermans D, et al. Hypotension during hemodialysis results from an impairment of arteriolar tone and left ventricular function. Clin Nephrol. 2005;63:276-83. doi: 10.5414/cnp63276

15. Prakash S, Garg AX, Heidenheim AP, House AA. Midodrine appears to be safe and effective for dialysis-induced hypotension: a systematic review. Nephrol Dial Transplant. 2004;19:2553-58. doi: 10.1093/ndt/gfh420.

16. Izcovich A, Gonzalez Malla C, Manzotti M, Catalano HN, Guyatt G. Midodrine for orthostatic hypotension and recurrent reflex syncope: A systematic review. Neurology 2014;83:1170-7. doi: 10.1212/WNL.0000000000000815.

17. Dheenan S, Venkatesan J, Grubb BP, Henrich WL. Effect of sertraline hydrochloride on dialysis hypotension. Am J Kidney Dis. 1998;31:624-30. doi: 10.1053/ajkd.1998.v31. pm9531178.

18. Yalcin AU, Sahin G, Erol M, Bal C. Sertraline hydrochloride treatment for patients with hemodialysis hypotension. Blood Purif. 2002;20:150-3. doi: 10.1159/000047001.

19. Molin C, Sakae TM, Schuelter-Trevisol F, Trevisol DJ. Effects of sertraline in the prevention of low blood pressure in patients undergoing hemodialysis. J Bras Nefrol 2019;41:492-500. doi: 10.1590/2175-8239-JBN-2018-0189.

20. Yalcin AU, Kudaiberdieva G, Sahin G, Gorenek B, Akcar N, Kuskus S, et al. Effect of sertraline hydrochloride on cardiac autonomic dysfunction in patients with hemodialysisinduced hypotension. Nephron Physiol. 2003;93:P21-8. doi: 10.1159/000066655.

21. Kudoh Y, Aoyama S, Torii T, Chen Q, Nagahara D, Sakata $\mathrm{H}$, et al. Hemodynamic stabilizing effects of L-carnitine in chronic hemodialysis patients. Cardiorenal Med. 2013;3:200-07. doi: 10.1159/000355016.

22. Ahmad S, Robertson HT, Golper TA, Wolfson M, Kurtin P, Katz LA, et al. Multicenter trial of L-carnitine in maintenance hemodialysis patients. II. Clinical and biochemical effects. Kidney Int. 1990;38:912-8. doi: 10.1038/ki.1990.290.

23. Lynch KE, Feldman HI, Berlin JA, Flory J, Rowan CG, Brunelli SM. Effects of L-carnitine on dialysis-related hypotension and muscle cramps: a meta-analysis. Am J Kidney Dis. 2008;52:962-71. doi: 10.1053/j. ajkd.2008.05.031.

24. Converse RL, Jacobsen TN, Jost C, Toto RD, Grayburn PA, Obregon TM, et al. Paradoxical withdrawal of reflex vasoconstriction as a cause of hemodialysis-induced hypotension. J Clin Invest. 1992;90:1657-65. doi: 10.1172/ JCI116037.

25. Kosinski D, Grubb BP, Temesy-Armos P. Pathophysiological aspects of neurocardiogenic syncope: current concepts and 
new perspectives. Pacing Clin Electrophysiol. 1995;18:71624. doi: 10.1111/j.1540-8159.1995.tb04666.x.

26. Grubb BP, Samoil D, Kosinski D, Wolfe D, Lorton M, Madu E. Fluoxetine hydrochloride for the treatment of severe refractory orthostatic hypotension. Am J Med. 1994;97:3668. doi: 10.1016/0002-9343(94)90304-2.

27. Grubb BP, Samoil D, Kosinskl D, Temesy-Armos P, Akpunonu B. The use of serotonin reuptake inhibitors for the treatment of recurrent syncope due to carotid sinus hypersensitivity unresponsive to dual chamber cardiac pacing. Pacing Clin Electrophysiol. 1994;17:1434-36. doi: 10.1111/j.1540-8159.1994.tb02463.x.

28. Grubb B. Serotonin and syncope: an emerging connection? Eur J Cardiac Pacing Electrophysiol. 1996;5:306-14.

29. Grubb B, Kosinski D. Preliminary observations on the use of venlafaxine hydrochloride in refractory orthostatic hypotension. J Serotonin Res. 1996;6:89-94.

30. Di Girolamo E, Di Iorio C, Sabatini P, Leonzio L, Barbone C, Barsotti A. Effects of paroxetine hydrochloride, a selective serotonin reuptake inhibitor, on refractory vasovagal syncope: a randomized, double-blind, placebo-controlled study. J Am Coll Cardiol. 1999;33:1227-30. doi: 10.1016/ s0735-1097(98)00694-9.

31. Chin CK, Roe CJ, Thomas GW. Fluoxetine: a treatment option for severe symptomatic postural hypotension in a diabetic haemodialysis patient. Aust N Z J Med. 1996;26:714. doi: 10.1111/j.1445-5994.1996.tb02948.x.

32. Atalay H, Solak Y, Biyik M, Biyik Z, Yeksan M, Uguz F, et al. Sertraline treatment is associated with an improvement in depression and health-related quality of life in chronic peritoneal dialysis patients. Int Urol Nephrol. 2010;42:52736. doi: 10.1007/s11255-009-9686-y.

33. Brewster UC, Ciampi MA, Abu-Alfa AK, Perazella MA. Addition of sertraline to other therapies to reduce dialysisassociated hypotension. Nephrology (Carlton). 2003;8:296301. doi: 10.1111/j.1440-1797.2003.00216.x.

34. Ibarra-Sifuentes HR, Del Cueto-Aguilera A, GallegosArguijo DA, Castillo-Torres SA, Vera-Pineda R, MartinezGranados RJ, et al. Levocarnitine decreases intradialytic hypotension episodes: a randomized controlled trial. Ther Apher Dial 2017;21:459-64. doi: 10.1111/1744-9987.12553.

Copyright $\odot 2020$ The Author(s); Published by Nickan Research Institute. This is an open-access article distributed under the terms of the Creative Commons Attribution License (http://creativecommons.org/licenses/by/4.0), which permits unrestricted use, distribution, and reproduction in any medium, provided the original work is properly cited. 